\title{
HARMLESS DELAYS IN A PERIODIC ECOSYSTEM
}

\author{
K. GOPALSAMY ${ }^{\prime}$
}

(Received 30 August 1980; revised 29 October 1982)

\begin{abstract}
Sufficient conditions are obtained for the existence of a unique linearly asymptotically stable positive periodıc solution of an ecosystem model of two species competition in a periodic environment with time lags in interspecific interactions. It is shown that if the self-regulating intraspecific interaction effects are strong enough and act without time delays then time delays of any length in the interspecific interactions cannot destabilise an otherwise stable ecosystem in a periodic environment.
\end{abstract}

\section{Introduction}

The purpose of this article is to establish a set of sufficient conditions for the existence of a unique linearly asymptotically stable positive periodic solution for a nonautonomous system of the form

$$
\begin{aligned}
& \frac{d x(t)}{d t}=x(t) f_{1}\left(t, x(t), y\left(t-\tau_{1}\right)\right), \\
& \frac{d y(t)}{d t}=y(t) f_{2}\left(t, x\left(t-\tau_{2}\right), y(t)\right) .
\end{aligned}
$$

In the context of population dynamics a model system of the form (1.1) means that the interspecific interactions occur with time delays $\tau_{1} \geqslant 0, \tau_{2} \geqslant 0$ in a periodic environment, if $f_{1}$ and $f_{2}$ are assumed to be periodic in $t$ with a common period $\omega \geqslant 0$.

Although model ecosystems in periodic environments have been investigated by a large number of authors (for some references see Gopalsamy [4], [5]), periodically varying environments in time delayed model ecosystems of two or more interacting species have not been investigated in the literature. The need for

\footnotetext{
' School of Mathematics, Flinders University, Bedford Park, S.A. 5042.

(c) Copyright Australian Mathematical Society 1984, Serial-fee code 0334-2700/84
} 
multispecies ecosystem models in periodic environments and time delayed interactions is evident from the extensive literature of that type relating to single species. While it is known that periodic ecosystems can support more species than uniform environments (Gopalsamy [4]), time delays might destabilise the system leading to a possible loss of one or more species. Furthermore, periodic environments in population dynamics have some relevance to natural selection and evolution; we will consider this aspect briefly at the end.

It will be an interesting and worthwhile investigation to examine the effects of time delays in the interspecific interactions in periodic environments; it is known that even in the case of constant environments such an investigation is more complicated. See for example Shibata and Saito [10] and Gopalsamy and Aggarwala [2] where it has been shown that such delays can lead to an onset of instability of steady states and Hopf-type bifurcation to oscillations including chaotic ones.

The principal finding of the following analysis of (1.1) is that, under suitable hypotheses on $f_{1}$ and $f_{2}$, the system (1.1) has a linearly asymptotically stable unique positive periodic solution for all nonnegative delays $\tau_{1}, \tau_{2}$ (while our numerical experiments suggest that the periodic solution of (1.1) is globally asymptotically stable and is independent of the delay parameters $\tau_{1}, \tau_{2}$ it is not known how one can prove such a strong result). Since time delays are usually associated with possible destabilization of otherwise stable systems, we can describe the time delays appearing on (1.1) as "harmless".

It has been recently shown by the author (Gopalsamy [6]) that if the self-regulating negative feedback in each species is strong enough and acts without time delays, then time delays of any length in the interspecific interactions cannot destabilise an otherwise stable ecosystem in a temporally uniform environment. The result of this paper is thus an extension to a periodic environment of the "harmless" nature of interspecific interaction delays in a temporally constant environment mentioned above.

\section{Existence and uniqueness of periodic solutions}

We will now formulate the problem of existence of periodic solutions of the periodic delay differential system

$$
\begin{aligned}
& \frac{d x_{1}(t)}{d t}=x_{1}(t) f_{1}\left(t, x_{1}(t), x_{2}\left(t-\tau_{12}\right)\right), \\
& \frac{d x_{2}(t)}{d t}=x_{2}(t) f_{2}\left(t, x_{1}\left(t-\tau_{21}\right), x_{2}(t)\right),
\end{aligned}
$$


(where $\tau_{12}, \tau_{21}$ are nonnegative constants) in a form suitable for the application of fixed point theory of positive operators. We make the following assumptions regarding $f_{1}$ and $f_{2}$ in (2.1).

$\left(\mathrm{H}_{1}\right)$ The partial (Fréchet) derivatives of $f_{1}, f_{2}$ satisfy the following:

$$
\frac{\partial f_{i}}{\partial x_{j}}<0, \quad i, j=1,2 \text { for all } t>-\tau \text { where } \tau=\max \left(\tau_{12}, \tau_{21}\right) .
$$

$\left(\mathrm{H}_{2}\right) f_{1}\left(t, x_{1}, x_{2}\right) \equiv f_{1}\left(t+\omega, x_{1}, x_{2}\right)$ for all $t>-\tau, x_{1} \geqslant 0, x_{2} \geqslant 0$.

$\left(\mathrm{H}_{3}\right)$ There exist positive constants $b_{t l}, b_{i}^{u}, a_{i l}, a_{i}^{u}(l=1,2)$ such that

$$
\begin{gathered}
b_{i l}-a_{i l} x_{t}<f_{i}\left(t, x_{1}, x_{2}\right)<b_{t}^{u}-a_{t}^{u} x_{i}, \\
b_{t l} / a_{a l}<b_{i}^{u} / a_{\imath}^{u},
\end{gathered}
$$

for $i=1,2$ and $\left(x_{1}, x_{2}\right) \in \mathbf{R}_{+} \times \mathbf{R}_{+}$. If $\tau=0$ then one can show that (2.1)-(2.4) will imply that the nondelayed periodic system (2.1) will have an asymptotically stable strictly positive periodic solution (for details see Gopalsamy [5]); this result will become a special case of what we obtain in the following.

We will use the following notation:

$$
\begin{aligned}
& \begin{array}{l}
B_{\omega}^{\tau}=\{h:[-\tau, \infty) \rightarrow \mathbf{R}, h \text { is continuous, } h(t+\omega)=h(t), t \geqslant-\tau\} \\
B=B_{\omega}^{\tau} \times B_{\omega}^{\tau},
\end{array} \\
& K_{1}=\left\{h \in B_{\omega}^{\tau} \mid h(t) \geqslant 0, t \in[-\tau, \infty)\right\} \\
& K=K_{1} \times K_{1}, \\
& \|h\|=\left\{\begin{array}{l}
\left.\sup |h(t)|, h \in B_{\omega}^{\tau}\right\} \\
t \in[0, \omega]
\end{array}\right\}, \\
& \left\|\left(x_{1}, x_{2}\right)\right\|=\max \left\{\left\|x_{1}\right\|,\left\|x_{2}\right\|,\left(x_{1}, x_{2}\right) \in B\right\} \\
& G_{1}=\left\{\left(x_{1}, x_{2}\right) \in K,\left\|\left(x_{1}, x_{2}\right)\right\| \leqslant b_{1}=\min \left(b_{11} / a_{1 l}, b_{2 l} / a_{2 l}\right)\right\} \\
& G_{2}=\left\{\left(x_{1}, x_{2}\right) \in K,\left\|\left(x_{1}, x_{2}\right)\right\| \leqslant b^{u}=\max \left(b_{1}^{u} / a_{1}^{u}, b_{2}^{u} / a_{2}^{u}\right)\right\}, \\
& \tilde{G}_{2}=\text { closure of } G_{2} \text { in } B, \text { and } \\
& \tilde{G}_{2} / G_{1}=\text { complement of } G_{1} \text { in } \tilde{G}_{2} ; \quad G_{1} \subset G_{2} \text { by }(2.4) .
\end{aligned}
$$

The cone $K=K_{1} \times K_{1}$ induces a partial order in $B$ and we will write $\left(x_{1}, x_{2}\right) \geqslant\left(y_{1}, y_{2}\right)$ if and only if $x_{1}-y_{1} \in K_{1}, x_{2}-y_{2} \in K_{1}$. We define an operator $N$ on $B$ as follows

$$
N\left(x_{1}, x_{2}\right)(t)=\left\{\begin{array}{l}
N_{1}\left(t, x_{1}, x_{2}\right) \\
N_{2}\left(t, x_{1}, x_{2}\right)
\end{array} \text { for } t>0\right.
$$


where

$$
\left.\begin{array}{rl}
N_{1}\left(t, x_{1}, x_{2}\right)= & \int_{0}^{\omega} G_{11}(m, \omega, t-s) g_{1}\left(m, s, x_{1}(x) x_{2}\left(s-\tau_{12}\right)\right) d s \\
& +\int_{0}^{t} G_{12}(m, 0, t-s) g_{1}\left(m, s, x_{1}(s), x_{2}\left(s-\tau_{12}\right)\right) d s, \\
N_{2}\left(t, x_{1}, x_{2}\right)= & \int_{0}^{\omega} G_{22}(m, \omega, t-s) g_{2}\left(m, s, x_{1}\left(s-\tau_{21}\right), x_{2}(s)\right) d s \\
& +\int_{0}^{t} G_{21}(m, 0, t-s) g_{2}\left(m, s, x_{1}\left(s-\tau_{21}\right), x_{2}(s)\right) d s,
\end{array}\right\}
$$

for $t>0$, with

$$
\begin{aligned}
& \left.g_{1}\left(m, s, x_{1}(s), x_{2}\left(s-\tau_{12}\right)\right)=m x_{1}(s)+x_{1}(s) f_{1}\left(s, x_{1}(s), x_{2}\left(s-\tau_{12}\right)\right),\right\} \\
& \left.g_{2}\left(m, s, x_{1}\left(s-\tau_{21}\right), x_{2}(s)\right)=m x_{2}(s)+x_{2}(s) f_{2}\left(s, x_{1}\left(s-\tau_{21}\right) x_{2},(s)\right),\right\} \\
& \left.\begin{array}{c}
G_{u}(m, \omega, t-s)=e^{-m \omega}\left(1-e^{-m \omega}\right)^{-1} e^{-m(t-s)}, \quad i=1,2, \\
G_{i j}(m, 0, t-s)=e^{-m(t-s)}, \quad i \neq j, i, j=1,2,
\end{array}\right\} \\
& N\left(x_{1}, x_{2}\right)(t)=\left\{\begin{array}{l}
N_{1}\left(n \omega+t, x_{1}, x_{2}\right) \\
N_{2}\left(n \omega+t, x_{1}, x_{2}\right)
\end{array} \text { for } t \in[-\tau, 0],\right.
\end{aligned}
$$

$m$ being a suitably selected positive constant (more will be said below) while $n$ is a positive constant such that $n \omega>\tau$.

We note immediately the following from the definition of $N$ : if $\left(x_{1}, x_{2}\right) \in$ $\tilde{G}_{2} / G_{1}$ then $N\left(x_{1}, x_{2}\right)(t) \geqslant 0$ and if $N\left(x_{1}, x_{2}\right)$ is periodic in $t$ of period $\omega$ for $t \geqslant 0$ then $N\left(x_{1}, x_{2}\right)$ is periodic in $t$ with period $\omega$ for $t \geqslant-\tau$. The existence of at least one positive periodic solution of (2.1)-(2.4) is established by means of a few lemmas and a result of Schmitt [9].

LEMMA 1. A necessary and sufficient condition for $\left(x_{1}(t), x_{2}(t)\right), t \geqslant-\tau$ to be a periodic solution of (2.1)-(2.4) of period $\omega$ is that $\left(x_{1}, x_{2}\right)$ be a fixed point of $N$ such that

$$
N\left(x_{1}, x_{2}\right)(t)=\left(x_{1}(t), x_{2}(t)\right) \text { and }\left(x_{1}, x_{2}\right) \in K \cap\left(\tilde{G}_{2} / G_{1}\right)
$$

Proof. The details of proof are similar to those of Lemma 2 of Busenberg and Cooke [1]. We first verify the continuity of $N\left(x_{1}, x_{2}\right)$ assuming that $N\left(x_{1}, x_{2}\right)(t)$ is periodic in $t$ of period $\omega$. The continuity of $N\left(x_{1}, x_{2}\right)$ for $t>0$ follows from the definition in (2.5)-(2.8) which also provides $\lim _{t \rightarrow 0+} N\left(x_{1}, x_{2}\right)(t)$. It will follow from (2.9) and the assumed periodicity of $N\left(x_{1}, x_{2}\right)$ that $N\left(x_{1}, x_{2}\right)$ is continuous 
for $t \geqslant-\tau$. Now we will verify the periodicity of $N\left(x_{1}, x_{2}\right)$ in $t$. A simple computation leads to

$$
N_{1}\left(t+\omega, x_{1}, x_{2}\right)-N_{1}\left(t, x_{1}, x_{2}\right)=e^{-m t} F_{1}(t),
$$

where

$$
\begin{aligned}
F_{1}(t)= & \int_{0}^{\omega} G_{11}(m, \omega, t-s) g_{1}\left(m, s, x_{1}(s), x_{2}\left(s-\tau_{12}\right)\right) d s \\
& +\int_{0}^{\omega} G_{12}(m, 0, t-s) g_{1}\left(m, s, x_{1}(s), x_{2}\left(s-\tau_{12}\right)\right) d s \\
& +\int_{\omega}^{\omega+t} G_{12}(m, 0, \omega-s) g_{1}\left(m, s, x_{1}(s), x_{2}\left(s-\tau_{12}\right)\right) d s \\
& -\int_{0}^{\omega} G_{11}(m, \omega,-s) g_{1}\left(m, s, x_{1}(s), x_{2}\left(s-\tau_{12}\right)\right) d s \\
& -\int_{0}^{t} G_{12}(m, 0,-s) g_{1}\left(m, s, x_{1}(s), x_{2}\left(s-\tau_{12}\right)\right) d s,
\end{aligned}
$$

which on simplification using the periodicity of $f_{1}$ shows that $F_{1}(t) \equiv 0$ whenever $x_{1}, x_{2} \in B$, thus implying the periodicity of $N_{1}$ in $t$ of period $\omega$. A similar argument for $N_{2}$ proves that $\left(x_{1}, x_{2}\right) \in B \Rightarrow N\left(x_{1}, x_{2}\right) \in B$.

Now suppose $\left(x_{1}, x_{2}\right)$ is a fixed point of $N$ and let $\left(x_{1}, x_{2}\right) \in K \cap\left(\tilde{G}_{2} / G_{1}\right)$. Then it is easily seen that $\left(x_{1}, x_{2}\right)$ is a solution of $(2.1)$ and since $\left(x_{1}, x_{2}\right) \in K \cap$ $\left(\tilde{G}_{2} / G_{1}\right)$ it will follow that $\left(x_{1}, x_{2}\right)$ is a periodic solution of $(2.1)$.

Conversely, let $\left(x_{1}, x_{2}\right)$ be a periodic solution of period $\omega$ of (2.1). Then $\left(x_{1}, x_{2}\right)$ is necessarily a periodic solution of

$$
\begin{aligned}
& \frac{d x_{1}(t)}{d t}+m x_{1}(t)=g_{1}\left(m, t, x_{1}(t), x_{2}\left(t-\tau_{12}\right)\right), \\
& \frac{d x_{2}(t)}{d t}+m x_{2}(t)=g_{2}\left(m, t, x_{1}\left(t-\tau_{21}\right), x_{2}(t)\right),
\end{aligned}
$$

for any arbitrary real constant $m$ and hence for any fixed constant $m>0$. It follows from (2.12) that any periodic solution of (2.1) and hence of (2.12) is a fixed point of $N\left(x_{1}, x_{2}\right)$. We claim that such a fixed point is in $K \cap\left(\tilde{G}_{2} / G_{1}\right)$. Suppose $\left(x_{1}, x_{2}\right) \notin K \cap\left(\tilde{G}_{2} / G_{1}\right)$. The following possibilities can arise:

(i) Either $x_{1}, x_{2}$ or both $x_{1}$ and $x_{2}$ may become negative for some value of $t$ and it will follow from (2.1) that subsequent values of $x_{1}, x_{2}$ or $x_{1}$ and $x_{2}$ will be negative and hence from (2.4) and (2.1) their derivatives will be negative for all subsequent $t$, implying that $x_{1}, x_{2}$ or $x_{1}$ and $x_{2}$ cannot be periodic.

(ii) While $\left(x_{1}, x_{2}\right) \in K \subset B$ there may be a value of $t$, say $t^{*}$, such that $x_{1}\left(t^{*}\right)>b_{1}^{u} / a_{1}^{u}$ or $x_{2}\left(t^{*}\right)>b_{1}^{u} / a_{1}^{u}$ and in such a case $x_{1}(t)$ or $x_{2}(t)$ cannot be periodic since respectively $d x_{1}\left(t^{*}\right) / d t<0$ or $d x_{2}\left(t^{*}\right) / d t<0$, which will imply that the values of $x_{1}\left(t^{*}\right)$ or $x_{2}\left(t^{*}\right)$ cannot be repeated from below. 
(iii) Similarly if there is a $t^{*}$ such that $0<x_{1}\left(t^{*}\right)<b_{1 l} / a_{1 l}$ or $0<x_{2}\left(t^{*}\right)<$ $b_{2 l} / a_{2 l}$ then such solutions cannot be periodic since $d x_{1}\left(t^{*}\right) / d t>0$ or $d x_{2}\left(t^{*}\right) / d t$ $>0$, which will mean that the values of $x_{1}\left(t^{*}\right)$ or $x_{2}\left(t^{*}\right)$ cannot be repeated from above.

Thus, every periodic solution of (2.1)-(2.4) is a fixed point of $N$ in $K \cap$ $\left(\tilde{G}_{2} / G_{1}\right) \subset B$.

Lemma 2. Let $N$ be as in (2.5)-(2.6). Suppose $\lambda \in(0,1)$ and $\lambda N\left(x_{1}, x_{2}\right)=$ $\left(x_{1}, x_{2}\right)$ for some $\left(x_{1}, x_{2}\right) \in K-\{0\}$. Then there exist $\alpha_{1}^{*}>0, \alpha_{2}^{*}>0$ such that

$$
\begin{aligned}
& 0<x_{1}(t) \leqslant \alpha_{1}^{*}<b_{1}^{u} / a_{1}^{u}, \\
& 0<x_{2}(t) \leqslant \alpha_{2}^{*}<b_{2}^{u} / a_{2}^{u},
\end{aligned}
$$

for $t \in[-\tau, \infty)$.

Proof. If $\lambda N\left(x_{1}, x_{2}\right)=\left(x_{1}, x_{2}\right)$ then from the definition of $N$ we get for all $t>0$ and $\left(x_{1}, x_{2}\right) \in K-\{0\}$,

$$
\begin{aligned}
& \frac{d x_{1}(t)}{d t}=\lambda x_{1}(t) f_{2}\left(t, x_{1}(t), x_{2}\left(t-\tau_{12}\right)\right)+x_{1}(t) m(\lambda-1), \\
& \frac{d x_{2}(t)}{d t}=\lambda x_{2}(t) f_{2}\left(t, x_{1}\left(t-\tau_{21}\right), x_{2}(t)\right)+x_{2}(t) m(\lambda-1) .
\end{aligned}
$$

For all $t \geqslant 0$ such that $x_{1}(t) \geqslant b_{1}^{u} / a_{1}^{u}$ or $x_{2}(t) \geqslant b_{2}^{u} / a_{2}^{u}$ we have $d x_{1} / d t<0$ or $d x_{2} / d t<0$; but these are impossible since $x_{1}, x_{2}$ are periodic in $t$. Thus by the continuity and periodicity of $x_{1}, x_{2}$ we have $x_{1}(t) \leqslant \alpha_{1}^{*}<b_{1}^{u} / a_{1}^{u}, x_{2}(t) \leqslant \alpha_{2}^{*}<$ $b_{2}^{u} / a_{2}^{u}$ for all $t \geqslant 0$; by the definition and periodicity the same will follow for all $t \geqslant-\tau$. A similar argument holds when $x_{1}(t)<0$ or $x_{2}(t)<0$, noting that in such a case $d x_{1} / d t>0$ or $d x_{2} / d t>0$, again contradicting the periodicity of $\left(x_{1}, x_{2}\right)$ in $t$.

Lemma 3. The operator $N$ maps $K \cap\left(\tilde{G}_{2} / G_{1}\right)$ into $K$ and is completely continuous.

Proof. From the definition of $N$ and (2.4) we derive that

$$
\begin{aligned}
N_{1}\left(t, x_{1}, x_{2}\right) \geqslant & \int_{0}^{\omega} G_{11}(m, \omega, t-s) x_{1}(s)\left[m+b_{1 /}-a_{1 /} x_{1}(s)\right] d s \\
& +\int_{0}^{t} G_{12}(m, 0, t-s) x_{1}(s)\left[m+b_{1 /}-a_{1 /} x_{1}(s)\right] d s .
\end{aligned}
$$

For $\left(x_{1}, x_{2}\right) \in K \cap\left(\tilde{G}_{2} / G_{1}\right)$, we have $0<x_{1}<b_{1}^{u} / a_{1}^{u}$ and hence we get $N_{1}\left(t, x_{1}, x_{2}\right)>0$ for all $t \geqslant 0$ by a proper choice of the positive constant say $m_{1}$. 
We make a similar choice say $m_{2}$ for $m$ for the case $N_{2}\left(t, x_{1}, x_{2}\right) \geqslant 0$ and then choose $m=\max \left(m_{1}, m_{2}\right)$. Thus we have

$$
N: K \cap\left(\tilde{G}_{2} / G_{1}\right) \rightarrow K \text { for } t \geqslant 0,
$$

and by the periodicity of $N$ we have the same for $t \geqslant-\tau$.

To prove the complete continuity of $N$ we proceed as follows. We know that if $\left(x_{1}, x_{2}\right) \in B$ then $N\left(x_{1}, x_{2}\right) \in B$ and so we can restrict $t$ to the set $[0, n \omega]$, $n \omega>\tau$. We write $N=L \circ Q$ where $L$ is the linear operator, $L: B \rightarrow B$,

$$
\begin{aligned}
& L\left(u_{1}, u_{2}\right)(t) \\
& \quad=\int_{0}^{\omega} G_{11}(m, \omega, t-s)\left[\begin{array}{l}
u_{1}(s) \\
u_{2}(s)
\end{array}\right] d s+\int_{0}^{t} G_{12}(m, 0, t-s)\left[\begin{array}{l}
u_{1}(s) \\
u_{2}(s)
\end{array}\right] d s,
\end{aligned}
$$

and $Q$ is the nonlinear operator

$$
\begin{gathered}
Q\left(u_{1}, u_{2}\right)(t)=\left\{\begin{array}{l}
g_{1}\left(m, t, u_{1}(t), u\left(t-\tau_{12}\right)\right) \\
g_{2}\left(m, t, u_{1}\left(t-\tau_{21}\right), u_{2}(t)\right)
\end{array} \text { for } t>0,\right. \\
Q\left(u_{1}, u_{2}\right)(t)=Q\left(u_{1}, u_{2}\right)(n \omega+t) \text { for } t \in[-\tau, 0]
\end{gathered}
$$

It can be found that

$$
\begin{gathered}
\left\|L\left(u_{1}, u_{2}\right)(t)\right\| \leqslant \max \left\{\frac{m+b_{1}^{u}}{m}, \frac{m+b_{2}^{u}}{m}\right\}\left\|\left(u_{1}, u_{2}\right)\right\|, \\
\left\|\frac{d}{d t} L\left(u_{1}, u_{2}\right)\right\| \leqslant 2 \max \left\{\frac{m+b_{1}^{u}}{m}, \frac{m+b_{2}^{u}}{m}\right\}\left\|\left(u_{1}, u_{2}\right)\right\| .
\end{gathered}
$$

The complete continuity of the linear map $L$ follows from (2.17). Now since $Q$ is continuous and maps bounded sets into bounded sets it will follow that $L \circ Q$ is completely continuous.

To prove the existence of periodic solutions of (2.1)-(2.4) we will use the following result of Schmitt [9].

THEOREM (Schmitt). Let $H_{1}$ and $H_{2}$ be nonempty bounded open neighbourhoods of the origin in a Banach space $B$ with a positive cone $K$ such that $H_{1} \subset H_{2}$ and let $N$ : $K \cap\left(\tilde{H}_{2} / H_{1}\right) \rightarrow K$ be completely continuous and which satisfies the following conditions:

(a) There exists $\left(z_{1}, z_{2}\right) \in K \subset B,\left\|\left(z_{1}, z_{2}\right)\right\|=1$ and $a \mu$ satisfying $\mu>$ $\sup \left\{\left\|\left(x_{1}, x_{2}\right)-N\left(y_{1}, y_{2}\right)\right\|,\left(x_{1}, x_{2}\right) \in K \cap H_{1},\left(y_{1}, y_{2}\right) \in K \cap \partial H_{2}\right\}$ such that all solutions $\left(x_{1}, x_{2}\right) \in K \cap\left(\tilde{H}_{2} / H_{1}\right)$ of $\left(x_{1}, x_{2}\right)=N\left(x_{1}, x_{2}\right)+\lambda\left(z_{1}, z_{2}\right)$ with $0<\lambda$ $<\mu$ satisfy $\left(x_{1}, x_{2}\right) \notin \partial H_{1}$. 
(b) All solutions $\left(x_{1}, x_{2}\right) \in K \cap\left(\tilde{H}_{2} / H_{1}\right)$ of $\left(x_{1}, x_{2}\right)=\lambda N\left(x_{1}, x_{2}\right), 0<\lambda<1$ satisfy $\left(x_{1}, x_{2}\right) \notin \partial H_{2}$.

Then $N$ has at least one fixed point in $K \cap\left(\tilde{H}_{2} / H_{1}\right)$.

We can now present our existence result.

THEOREM 1. Assume that $f_{1}, f_{2}$ of (2.1) satisfy (2.2)-(2.4). Then there exists at least one positive periodic solution of (2.1)-(2.4).

Proof. By Lemma 1, it is enough to show that the operator $N: K \cap\left(\tilde{G}_{2} / G_{1}\right) \rightarrow$ $K \subset B$ has at least one fixed point in $\tilde{G}_{2} / G_{1}$ and we will do this by applying the result of Schmitt [9]. First we let

$$
\begin{aligned}
& H_{2}=\left\{\left(x_{1}, x_{2}\right) \in B\left\|\mid\left(x_{1}, x_{2}\right)\right\|<\max \left(b_{1}^{u} / a_{1}^{u}, b_{2}^{u} / a_{2}^{u}\right)\right\} \equiv G_{2}, \\
& H_{1}=\left\{\left(x_{1}, x_{2}\right) \in B\left\|\left(x_{1}, x_{2}\right)\right\|<\min \left(b_{1 /} / a_{1 l}, b_{1} / a_{1 l}\right)\right\} \equiv G_{1} .
\end{aligned}
$$

By Lemma 3, $N: K \cap\left(\tilde{H}_{2} / H_{1}\right) \rightarrow K$ is completely continuous. Choose $\mu=\infty$ and $\left(z_{1}, z_{2}\right) \equiv(1,1)$. Then all solutions $\left(x_{1}, x_{2}\right) \in K \cap\left(\tilde{H}_{2} / H_{1}\right)$ of $\left(x_{1}, x_{2}\right)=$ $N\left(x_{1}, x_{2}\right)+\lambda\left(z_{1}, z_{2}\right), 0<\lambda<\infty$, satisfy (2.1) and hence by their periodicity $\left(x_{1}, x_{2}\right) \notin \partial H_{1}$. The condition (a) of Schmitt's theorem is satisfied since $\mu=\infty$ by choice and $\left(x_{1}, x_{2}\right)$ and $\left(z_{1}, z_{2}\right)$ are periodic. By Lemma 2 the condition (b) of Schmitt's theorem is satisfied. Thus there exists at least one fixed point of $N$ in $K \cap\left(\tilde{H}_{2} / H_{1}\right)$ and hence there exists at least one positive periodic solution of (2.1)-(2.4) and the proof is complete.

We remark that so far no restriction on the magnitudes of the delays $\tau_{12}, \tau_{21}$ has been imposed except that they be nonnegative.

\section{Uniqueness of the periodic solution}

Applications of fixed point theorems to prove the existence of solutions of nonlinear problems usually suffer from the drawback that the uniqueness of solutions has to be proved often by other methods. We will use the following result of Smith [11] which is a slight variation of his Theorem 3.5 (see also Busenberg and Cooke [1]).

THEOREM (Smith). Let $N$ be an operator on a cone $K$ in the Banach space $B$ and let $K^{*}=\left\{\left(x_{1}, x_{2}\right) \in K \cap B, 0 \leqslant\left\|\left(x_{1}, x_{2}\right)\right\| \leqslant C^{*}\right\}$ where $C^{*}$ is some positive constant. Assume the following: 
(a) If $\left(x_{1}, x_{2}\right) \in K^{*}-\{(0,0)\}$ and $N\left(x_{1}, x_{2}\right)=\left(x_{1}, x_{2}\right)$ then $\left(x_{1}, x_{2}\right) \in$ interior of $K^{*}$.

(b) $N$ is monotonic nondecreasing on $K^{*}$.

(c) If $\left(x_{1}, x_{2}\right)$ is in the interior of $K^{*}$ and if $0<\lambda<1$ then there exists an $\eta>0$ depending on $\left(x_{1}, x_{2}\right)$ and $\lambda$ such that $N\left(\lambda x_{1}, \lambda x_{2}\right) \geqslant \lambda(1+\eta) N\left(x_{1}, x_{2}\right)$. Then $N$ has at most one fixed point different from $(0,0)$ in $K^{*} \subset B$.

The cone $K=K_{1} \times K_{1}$ where $K_{1}=\left\{h \in B_{\omega}^{\tau}: h(t) \geqslant 0, t \in[-\tau, \infty)\right\}$ is slightly too wide in that solutions of the form $\left\{x_{1}(t), 0\right\}$ and $\left\{0, x_{2}(t)\right\}$ for (2.1) are also possible. We choose a narrower cone $K^{*}$ in $B$ as follows: define

$$
S=\left\{\left(x_{1}, x_{2}\right) \in B \mid b_{1 /} / a_{1 \prime} \leqslant\left\|x_{1}\right\| \leqslant b_{1}^{u} / a_{1}^{u}, b_{2 l} / a_{2 l} \leqslant\left\|x_{2}\right\| \leqslant b_{2}^{u} / a_{2}^{u}\right\},
$$

and let

$$
K^{*}=\left\{\lambda\left(x_{1}, x_{2}\right) \in B \mid\left(x_{1}, x_{2}\right) \in S, \lambda \geqslant 0\right\}
$$

Since $\tilde{G}_{2} / G_{1} \subset K^{*}$, it is not difficult to see that $N: \tilde{G}_{2} / G_{1} \rightarrow B$ has at least one fixed point which is a periodic solution of (2.1)-(2.4). We will prove that this solution is unique by applying the result of Smith cited above. The following lemmas are needed first.

LEMMA 4. If $\left(x_{1}, x_{2}\right) \in K^{*}-\{0,0\}$ and if $N\left(x_{1}, x_{2}\right)=\left(x_{1}, x_{2}\right)$ then $\left(x_{1}, x_{2}\right)$ $\in$ interior of $K^{*}$.

Proof. If $\left(x_{1}, x_{2}\right)=N\left(x_{1}, x_{2}\right)$ and $\left(x_{1}, x_{2}\right) \in K^{*}-\{0,0\}$, then $\left(x_{1}, x_{2}\right)$ is a periodic solution of (2.1)-(2.4) and hence we have

$$
\begin{aligned}
& \left(b_{1 l}-a_{1 /} x_{1}\right) x_{1}<\frac{d x_{1}}{d t}<\left(b_{1}^{u}-a_{1}^{u} x_{1}\right) x_{1}, \\
& \left(b_{2 l}-a_{2 l} x_{2}\right) x_{2}<\frac{d x_{2}}{d t}<\left(b_{2}^{u}-a_{2}^{u} x_{2}\right) x_{2},
\end{aligned}
$$

and hence any nonzero periodic solution $\left(x_{1}, x_{2}\right)$ of $(2.1)-(2.4)$ has to be such that

$$
\begin{aligned}
& b_{11} / a_{1 l}<x_{1}(t)<b_{1}^{u} / a_{1}^{u}, \\
& b_{21} / a_{21}<x_{2}(t)<b_{2}^{u} / a_{2}^{u},
\end{aligned}
$$

for $t>-\tau$, which proves the lemma.

Lemma 5. The mapping $N$ defined by (2.5)-(2.9) is monotone nondecreasing on $K^{*}$. 
Proof. Let $\left(x_{1}, x_{2}\right) \in K^{*},\left(y_{1}, y_{2}\right) \in K^{*}$ such that $\left(x_{1}-y_{1}, x_{2}-y_{2}\right) \in K^{*}$ and $\left(x_{1}, x_{2}\right) \neq\left(y_{1}, y_{2}\right)$. Then $x_{1} \geqslant y_{1}, x_{2} \geqslant y_{2}$. From the definition of $N$ we derive that

$$
\begin{aligned}
& N_{1}\left(t, x_{1}, x_{2}\right)-N_{1}\left(t, y_{1}, y_{2}\right) \\
& =\int_{0}^{\omega} G_{11}(m, \omega, t-s)\left\{m\left[x_{1}(s)-y_{1}(s)\right]+x_{1}(s) f_{1}\left(s, x_{1}(s), x_{2}\left(s-\tau_{12}\right)\right)\right. \\
& \left.-y_{1}(s) f_{1}\left(s, y_{1}(s), y_{2}\left(s-\tau_{12}\right)\right)\right\} d s \\
& +\int_{0}^{t} G_{12}(m, 0, t-s) \\
& \quad \times\left\{m\left[x_{1}(s)-y_{1}(s)\right]+\left[x_{1}(s) f_{1}\left(s, x_{1}(s), x_{2}\left(s-\tau_{12}\right)\right)\right.\right. \\
& \left.\left.\quad-y_{1}(s) f_{1}\left(s, y_{1}(s), y_{2}\left(s-\tau_{12}\right)\right)\right]\right\} d s,
\end{aligned}
$$

with a similar relation for $N_{2}\left(t, x_{1}, x_{2}\right)-N_{2}\left(t, y_{1}, y_{2}\right)$. Since $f_{1}$ is continuous, $f$ is bounded on $K^{*}$; also it will follow from $\left(x_{1}, x_{2}\right) \neq\left(y_{1}, y_{2}\right)$ that

$$
0 \approx x_{1}(s)-y_{1}(s) \geqslant 0 \text { and } 0 \neq x_{2}(s)-y_{2}(s) \geqslant 0 \text {; }
$$

a sufficiently large positive constant $m$ will make the right side of (3.4) positive. A similar argument will lead to $N_{2}\left(t, x_{1}, x_{2}\right)-N_{2}\left(t, y_{1}, y_{2}\right)>0$ perhaps with a larger constant $m$. Finally we can choose the bigger of these two constants $m$ to yield

$$
N\left(x_{1}, x_{2}\right)-N\left(y_{1}, y_{2}\right) \geqslant 0 \text { for }\left(x_{1}-y_{1}, x_{2}-y_{2}\right) \in K^{*}-\{(0,0)\} .
$$

The monotonicity of $N$ on $K^{*}$ follows.

Lemma 6. Let $\left(x_{1}, x_{2}\right) \in$ interior of $K^{*}$ and let $\lambda$ be a constant such that $0<\lambda<1$. Suppose $f_{1}, f_{2}$ satisfy (2.2)-(2.4). Then there exists a positive constant $\eta$ such that

$$
N\left(\lambda x_{1}, \lambda x_{2}\right) \geqslant \lambda(1+\eta) N\left(x_{1}, x_{2}\right)
$$

Proof. A direct computation leads to

$$
\begin{aligned}
& N_{1}\left(t, \lambda x_{1}, \lambda x_{2}\right)-\lambda N_{1}\left(t, x_{1}, x_{2}\right) \\
&= \lambda \int_{0}^{\omega} G_{11}(m, \omega, t-s) g_{1}\left(s, x_{1}(s), x_{2}\left(s-\tau_{12}\right)\right) H_{1}\left(s, \lambda, x_{1}, x_{2}\right) d s \\
&+\lambda \int_{0}^{t} G_{11}(m, 0, t-s) g_{1}\left(s, x_{1}(s), x_{2}\left(s-\tau_{12}\right)\right) H_{1}\left(s, \lambda, x_{1}, x_{2}\right) d s,
\end{aligned}
$$


where

$$
H_{1}\left(s, \lambda, x_{1}, x_{2}\right)=\frac{m x_{1}(s)+x_{1}(s) f_{1}\left(s, \lambda x_{1}(s), \lambda x_{2}\left(s-\tau_{12}\right)\right)}{m x_{1}(s)+x_{1}(s) f_{1}\left(s, x_{1}(s), x_{2}\left(s-\tau_{12}\right)\right)}-1 .
$$

Since $f_{1}$ is decreasing (see (2.2)) with respect to $x_{1}$ and $x_{2}$ there exists a positive constant say $\eta_{1}$ such that

$$
H_{1}\left(s, \lambda, x_{1}, x_{2}\right) \geqslant \eta_{1} \quad \text { for } 0<\lambda<1, s>0,\left(x_{1}, x_{2}\right) \in K^{*},
$$

and hence

$$
N_{1}\left(t, \lambda x_{1}, \lambda x_{2}\right) \geqslant \lambda\left(1+\eta_{1}\right) N_{1}\left(t, x_{1}, x_{2}\right) .
$$

A similar argument will show that there exists an $\eta_{2}>0$ such that

$$
N_{2}\left(t, \lambda x_{1}, \lambda x_{2}\right) \geqslant \lambda\left(1+\eta_{2}\right) N_{2}\left(t, x_{1}, x_{2}\right),
$$

and the result follows if we choose $\eta=\min \left(\eta_{1}, \eta_{2}\right)$.

Now the following uniqueness result is a direct consequence of the result of Smith [11] and the Lemmas 4, 5, 6 .

THEOREM 2. Suppose $f_{1}, f_{2}$ of (2.1) satisfy (2.2)-(2.4). Then there exists a unique $\omega$-periodic strictly positive periodic solution of $(2.1)$.

\section{Linear stability of the periodic solution}

Let $\left\{x_{1}^{*}(t), x_{2}^{*}(t), t \geqslant-\tau\right\}$ be the strictly positive unique periodic solution of (2.1). Let $\left\{x_{1}(t), x_{2}(t), t \geqslant-\tau\right\}$ be any other solution of (2.1). To study the linear stability of $\left(x_{1}^{*}, x_{2}^{*}\right)$ we let

$$
\begin{aligned}
& x_{1}(t) \equiv x_{1}^{*}(t)\left\{1+X_{1}(t)\right\} \\
& x_{2}(t) \equiv x_{2}^{*}(t)\left\{1+X_{2}(t)\right\}
\end{aligned}
$$

and derive that the perturbations $\left(X_{1}, X_{2}\right)$ are governed by the linear variational system (after neglecting the nonlinear terms in $X_{1}, X_{2}$ )

$$
\begin{aligned}
& \frac{d X_{1}(t)}{d t}=-a_{11}(t) x_{1}^{*}(t) X_{1}(t)-a_{12}(t) x_{2}^{*}\left(t-\tau_{12}\right) X_{2}\left(t-\tau_{12}\right), \\
& \frac{d X_{2}(t)}{d t}=-a_{21}(t) x_{1}^{*}\left(t-\tau_{21}\right) X_{1}\left(t-\tau_{21}\right)-a_{22}(t) x_{2}^{*}(t) X_{2}(t),
\end{aligned}
$$


for $t>0$, where

$$
\left.\begin{array}{l}
-a_{11}(t)=\frac{\partial f_{1}}{\partial x_{1}} \\
-a_{12}(t)=\frac{\partial f_{1}}{\partial x_{2}}
\end{array}\right\} \quad \text { evaluated at }\left(t, x_{1}^{*}(t), x_{2}^{*}\left(t-\tau_{12}\right)\right),
$$

To establish the linear asymptotic stability of $\left(x_{1}^{*}(t), x_{2}^{*}(t)\right)$ it is enough to prove that of the trivial solution of (4.2)-(4.3). We will do this by means of a combination of a comparison differential system and a Lyapunov function. First we define

$$
\frac{d\left|X_{i}(t)\right|}{d t}=\left\{\begin{array}{cl}
\frac{d X_{i}(t)}{d t} & \text { if } X_{i}(t) \geqslant 0, \\
-\frac{d X_{t}(t)}{d t} & \text { if } X_{i}(t)<0,
\end{array} \quad i=1,2\right.
$$

and derive from (4.2)-(4.3) the following comparison system of differential inequalities:

$$
\begin{aligned}
& \frac{d\left|X_{1}(t)\right|}{d t} \leqslant-b_{11} x_{1}^{*}(t)\left|X_{1}(t)\right|+b_{12} x_{2}^{*}\left(t-\tau_{12}\right)\left|X_{2}\left(t-\tau_{12}\right)\right|, \\
& \frac{d\left|X_{2}(t)\right|}{d t} \leqslant b_{21} x_{1}^{*}\left(t-\tau_{21}\right)\left|X_{1}\left(t-\tau_{22}\right)\right|-b_{22} x_{2}^{*}(t)\left|X_{2}(t)\right|,
\end{aligned}
$$

for $t>0$, where

$$
\begin{aligned}
-b_{11} & =\max _{t \geqslant-\tau}\left\{-a_{11}(t)\right\}, \\
b_{12} & =\left|\min _{t \geqslant-\tau}\left\{-a_{12}(t)\right\}\right|, \\
b_{21} & =\left|\min _{t \geqslant-\tau}\left\{-a_{21}(t)\right\}\right|, \\
-b_{22} & =\max _{t \geqslant-\tau}\left\{-a_{22}(t)\right\} .
\end{aligned}
$$

We can now formulate the result as follows. By the periodicity of $a_{i j}$, the constants $b_{i}$, are independent of $\tau$ (see also (2.2)). 
THEOREM 3. The unique strictly positive periodic solution $\left\{x_{1}^{*}(t), x_{2}^{*}(t), t \geqslant-\tau\right\}$ of (2.1)-(2.4) is linearly asymptotically stable provided

$$
\left|-b_{11}\right|>b_{21} \text { and }\left|-b_{22}\right|>b_{12} \text {, }
$$

where the constants $b_{i j}(i, j=1,2)$ are as defined in (4.6).

Proof. Consider the scalar valued Lyapunov-like function $v(t)=V\left(t, X_{1}, X_{2}\right)$ where

$$
\begin{aligned}
v(t)=V\left(t, X_{1}, X_{2}\right)= & \left|X_{1}(t)\right|+\left|X_{2}(t)\right|+\int_{t-\tau_{12}}^{t} b_{12} x_{2}^{*}(u)\left|X_{2}(u)\right| d u \\
& +\int_{t-\tau_{21}}^{t} b_{21} x_{1}^{*}(u)\left|X_{1}(u)\right| d u .
\end{aligned}
$$

Since $x_{1}^{*}, x_{2}^{*}$ are strictly positive, it is found from (4.7)-(4.8) that

$$
\frac{d v}{d t} \leqslant-\left[\left(\left|b_{11}\right|-b_{21}\right) x_{1}^{*}(t)\left|X_{1}(t)\right|+\left(\left|b_{22}\right|-b_{22}\right) x_{2}^{*}(t)\left|X_{2}(t)\right|\right],
$$

for $t>0$. It follows from (4.7)-(4.9) that $V\left(t, X_{1}, X_{2}\right) \geqslant\left|X_{1}(t)\right|+\left|X_{2}(t)\right|$ and $d v / d t \leqslant 0$, which will imply the stability of the trivial solution of (4.2)-(4.3). We claim that (4.9) also implies the asymptotic stability of the trivial solution of (4.2)-(4.3) in the sense that

$$
\lim _{t \rightarrow \infty}\left[\left|X_{1}(t)\right|+\left|X_{2}(t)\right|\right]=0
$$

for all bounded initial conditions $X_{1}(s), X_{2}(s), s \in[-\tau, 0]$. Suppose (4.10) is not true. Then we can find an increasing sequence $\left\{t_{m}\right\} \rightarrow \infty$ as $m \rightarrow \infty$ such that

$$
\begin{array}{r}
\left(\left|b_{11}\right|-b_{21}\right) x_{1}^{*}\left(t_{m}\right)\left|X_{1}\left(t_{m}\right)\right|+\left(\left|b_{22}\right|-b_{12}\right) x_{2}^{*}\left(t_{m}\right)\left|X_{2}\left(t_{m}\right)\right|>\varepsilon, \\
m=1,2,3, \ldots
\end{array}
$$

Using the right derivative $d v / d t$ of $v$ we derive that

$$
v\left(t_{m}-\varepsilon\right)-v\left(t_{m}\right) \geqslant-\int_{t_{m}-\varepsilon}^{t_{m}} \frac{d v(\tau)}{d \tau} d \tau .
$$

If $\varepsilon$ is small enough we have from (4.9), (4.11), (4.12) that $d v(\tau) / d \tau<(-\varepsilon / 2)$ and $v\left(t_{m}-\varepsilon\right)-v\left(t_{m}\right) \geqslant \varepsilon^{2} / 2$ and hence

$$
\begin{aligned}
v\left(t_{m}\right) & \leqslant v\left(t_{m}-\varepsilon\right)-\left(\varepsilon^{2} / 2\right) \\
& \leqslant v\left(t_{m-1}\right)-\left(\varepsilon^{2} / 2\right) \\
& \leqslant v\left(t_{m-2}\right)-2\left(\varepsilon^{2} / 2\right) \\
\vdots & \\
& \leqslant v\left(t_{0}\right)-m\left(\varepsilon^{2} / 2\right) .
\end{aligned}
$$


Thus for sufficiently large $m, v\left(t_{m}\right)$ becomes negative which contradicts the nonnegative nature of $v$ for $t \geqslant 0$. This contradiction shows that $\left|X_{1}(t)\right|+$ $\left|X_{2}(t)\right| \rightarrow 0$ as $t \rightarrow \infty$ and the proof is complete.

As an example let us consider the case of two species Lotka-Volterra type competition in a periodic environment for which we have

$$
\begin{gathered}
f_{1}\left(t, x_{1}(t), x_{2}\left(t-\tau_{12}\right)\right)=1-\left\{\frac{x_{1}(t)+\alpha x_{2}\left(t-\tau_{12}\right)}{K_{1}(t)}\right\}, \\
f_{2}\left(t, x_{1}\left(t-{ }_{21}\right), x_{2}(t)\right)=1-\left\{\frac{\beta x_{1}\left(t-\tau_{21}\right)+x_{2}(t)}{K_{2}(t)}\right\},
\end{gathered}
$$

where $\alpha, \beta$ are positive constants and $K_{1}, K_{2}$ are strictly positive continuous periodic functions with a common period. Equations (4.6) and (4.7) for this special case lead to

$$
\begin{aligned}
& \left|\max _{t \geq-\tau}\left\{-a_{11}(t)\right\}\right|=\max _{t \geq-\tau}\left(1 / K_{1}(t)\right)>\left|\min _{t \geq-\tau}\left(-a_{21}(t)\right)\right|=\min _{t \geq-\tau}\left(\beta / K_{2}(t)\right), \\
& \left|\max _{t \geq-\tau}\left\{-a_{22}(t)\right\}\right|=\max _{t \geq-\tau}\left(1 / K_{2}(t)\right)>\left|\min _{t \geq-\tau}\left(-a_{12}(t)\right)\right|=\min _{t \geq-\tau}\left(\alpha / K_{1}(t)\right) .
\end{aligned}
$$

The inequalities reduce in the absence of temporal variations in $K_{1}, K_{2}$ to the inequality $0<\alpha \beta<1$, which is well known for the global asymptotic stability of the positive steady state corresponding to the above example in a constant environment. The above example shows also that delays in interspecific interactions have no influence on the local asymptotic stability of the unique periodic solution of the periodic ecosystem. A number of cases of this type in a constant environment have been established recently by the author (Gopalsamy [6]). A numerical example is illustrated at the end.

\section{Some ecological and evolutionary consequences}

It can be found that all the conclusions on the existence, uniqueness and stability of a periodic solution of (2.1)-(2.4) are valid when $\tau_{12}=0$ or $\tau_{21}=0$ or both $\tau_{12}=0$ and $\tau_{21}=0$; thus it will follow that delays in interspecific competition do not destabilise an otherwise stable coexistence (oscillatory or nonoscillatory) of two competing species. In forthcoming work we will extend the analysis of the system (2.1)-(2.4) to a system of $n$-competing species with a hypothesis similar to that of (2.4). It is however an open problem to investigate the effects of delays in intraspecific competition in a periodic environment. 
Pianka ([8], pages 139-140) remarks that "In real populations, rates of increase, competitive abilities and carrying capacities do vary from individual to individual, with population density in space and time. Indeed temporal variation in the environment may often allow coexistence by continually altering the competitive abilities of populations inhabiting it. Moreover time lags are doubtlessly of some importance in real populations. Finally a heterogeneous environment may allow real competitors to evolve divergent resource utilization patterns and to reduce interspecific competitive inhibition."

To understand the mechanism of selection pressure against competitive inhibition we first write the Malthusian parameters $\mu_{1}, \mu_{2}$ of fitness for the two species as follows (see MacArthur [7], pages 226-230):

$$
\begin{aligned}
& \mu_{1}=\frac{1}{x_{1}(t)} \frac{d x_{1}(t)}{d t}=f_{1}\left(t, x_{1}(t), x_{2}\left(t-\tau_{12}\right)\right), \\
& \mu_{2}=\frac{1}{x_{2}(t)} \frac{d x_{2}(t)}{d t}=f_{2}\left(t, x_{1}\left(t-\tau_{21}\right), x_{2}(t)\right) .
\end{aligned}
$$

Since $\left(x_{1}(t), x_{2}(t)\right) \rightarrow\left(x_{1}^{*}(t), x_{2}^{*}(t)\right)$ for large $t$ we can write the parameters of fitness in the form

$$
\begin{aligned}
& \mu_{1}=f_{1}\left(t, x_{1}^{*}(t), x_{2}^{*}\left(t-\tau_{12}\right)\right), \\
& \mu_{2}=f_{2}\left(t, x_{1}^{*}\left(t-\tau_{21}\right), x_{2}^{*}(t)\right) .
\end{aligned}
$$

In these relations we can use

$$
\begin{aligned}
& x_{2}^{*}\left(t-\tau_{12}\right)=x_{2}^{*}(t)-\tau_{12} \frac{d x_{2}^{*}\left(t-\tau_{12}+\theta_{1} \tau_{12}\right)}{d t}, \\
& x_{1}^{*}\left(t-\tau_{21}\right)=x_{1}^{*}(t)-\tau_{21} \frac{d x_{1}^{*}\left(t-\tau_{21}+\theta_{2} \tau_{21}\right)}{d t},
\end{aligned}
$$

$\theta_{1}, \theta_{2} \in(0,1)$, and rewrite $\mu_{1}, \mu_{2}$ in the form

$$
\begin{aligned}
& \mu_{1}=f_{1}\left(t, x_{1}^{*}(t), x_{2}^{*}(t)-\tau_{12} \frac{d x_{2}^{*}\left(t-\tau_{12}+\theta_{1} \tau_{12}\right)}{d t}\right), \\
& \mu_{2}=f_{2}\left(t, x_{1}^{*}(t)-\tau_{21} \frac{d x_{1}^{*}\left(t-\tau_{21}+\theta_{2} \tau_{21}\right)}{d t}, x_{2}^{*}(t)\right),
\end{aligned}
$$

$\theta_{1}, \theta_{2} \in(0,1)$.

It is seen that the presence of the delays $\tau_{12}$ and $\tau_{21}$ provides additional sources of variability in the fitness parameters and thereby in the selection pressure against interspecific competition. Since a necessary and sufficient condition for natural selection to act is differential (variable) rates of population growth, we find that time delays coupled with periodicity of an environment may allow real competitors to evolve divergent resource utilization patterns and to reduce 




Frgure 1. Convergence to oscillations for $\mathrm{T}=3$.



FIGURE 2. Convergence to oscillations for $\tau=10$.

interspecific competitive inhibition. For some remarks relating to the significance of a periodic environment to sustain the coexistence of a large number of competing species in the absence of delays, we refer to a recent article (Gopalsamy [4]). 
The following example has been numerically solved and the solutions are displayed graphically for two different values of the delay parameter $\tau$ :

$$
\begin{gathered}
\frac{d x_{1}(t)}{d t}=x_{1}(t)\left[1-x_{1}(t)+0.4 x_{2}(t-\tau) / K_{1}(t)\right] \\
\frac{d x_{2}(t)}{d t}=x_{2}(t)\left[1-\left\{0.8 x_{1}(t-\tau)+x_{2}(t)\right\} / K_{2}(t)\right]
\end{gathered}
$$

where $K_{1}(t)=2.0+\sin (\pi t)$ and $K_{2}(t)=2.5+\sin (\pi t)$.

\section{References}

[1] S. Busenberg and K. L. Cooke, "Periodic solutions of a periodic nonlinear delay differential equation," SIAM J. Appl. Math. 34 (1978), 704-721.

[2] K. Gopalsamy and B. D. Aggarwala, "Limıt cycles in two species competition with time delays", J. Austral. Math. Soc. Ser. B 22 (1980), 148-160.

[3] K. Gopalsamy. "Time lags and global stability in two species competition", Bull. Math. Bıl 42 (1980), 729-737.

[4] K. Gopalsamy, "Limit cycles in periodically perturbed population systems", Bull. Math. Biol. 43 (1981), 463-485.

[5] K. Gopalsamy, "Exchange of equilibria in two species Lotka-Volterra competition", J. Austral. Math. Soc Ser. B 24 (1982), 160-170.

[6] K. Gopalsamy, "Harmless delays in model systems", Bull. Math. Biol. (in press).

[7] R. MacArthur, Geographical ecology (Harper and Row, New York, 1972).

[8] E. R. Pianka, Evolutionary ecology (Harper and Row, New York, 1974).

[9] K. Schmitt, "Fixed points and coincidence theorems with applications to nonlinear differential and integral equations", Rapp. \#97, Univ. Catholique de Louvain; Institut de Mathématique Pure et Appliquée, 1976.

[10] A. Shibata and N. Saito, "Time delays and chaos in two competing species", Math. Biosci. 51 (1980), 199-211.

[11] H. Smith, "On periodic solutions of delay integral equations modelling epidemics and population growth", Ph.D. Dissertation, University of Iowa, 1976. 